\title{
Estudo comparativo das estimulações ventricular direita e biventricular no pós-operatório de revascularização miocárdica
}

\author{
Luciano C. ALBUQUERQUE*, João R. SANT'ANNA**, Alcides J. ZAGO***, \\ Flávio J.P. VELHO*, João Batista PETRACCO*
}

RBCCV 44205-573

\begin{abstract}
Albuquerque L C, Sant'anna J R, Zago A J, Velho F J P, Petracco J B - Estudo comparativo das estimulações ventricular direita e biventricular no pós-operatório de revascularização miocárdica. Rev Bras Cir Cardiovasc 2002; 17(1): 61-72.
\end{abstract}

RESUMO: Objetivo: Nos anos recentes, a ressincronização ventricular tem sido proposta como adjuvante no tratamento da insuficiência cardíaca congestiva. O objetivo deste estudo é comparar as alterações eletrocardiográficas e o efeito hemodinâmico imediato das estimulações ventricular direita (EVD) e biventricular (EBV), no pós-operatório de operação de revascularização miocárdica (CRM) com circulação extracorpórea (CEC).

Casuística e Métodos: Em um ensaio clínico cruzado, 13 pacientes com doença coronária multiarterial, e fração de ejeção inferior a $50 \%$, foram submetidos a estimulação epicárdica temporária univentricular direita e biventricular, no $5^{\circ}$ dia de pós-operatório. As variáveis analisadas foram duração do complexo QRS, dimensões do átrio esquerdo ( $A E$ ) e ventrículo esquerdo (VE), fração de encurtamento do VE (delta D) e fração de ejeção do VE. Os grupos foram comparados através do teste de t de Student para amostras pareadas, considerando-se nível de significância de 0,05.

Resultados: A duração média do complexo QRS foi de $185 \pm 26$ ms durante a EVD, e de $126 \pm 37$ $\mathrm{ms}$ com a EBV $(p<0,001)$. O diâmetro médio do AE com a EVD foi de $40 \pm 4 \mathrm{~mm}$, e de $35 \pm 4 \mathrm{~mm}$ na EBV $(p<0,001)$. As médias dos diâmetros diastólico e sistólico finais do VE foram, respectivamente, de $49 \pm 13 \mathrm{~mm}$ e $59 \pm 11 \mathrm{~mm}$ com a EVD, e de $42 \pm 12 \mathrm{~mm}$ e $52 \pm 10 \mathrm{~mm}$ durante a EBV $(p<0,001)$. A delta $D$ média do VE determinada pela EVD foi de $18 \pm 7 \%$, e de $22 \pm 8 \%$ com a $E B V(p=0,017)$. A fração de ejeção média do VE com a EVD foi de $33 \pm 14 \%$, e de $46 \pm 17 \%$ durante a EBV $(p<0,001)$.

Conclusão: No modelo estudado, a estimulação biventricular temporária determinou melhora significativa do desempenho hemodinâmico, em comparação à estimulação ventricular direita, e um complexo QRS com duração próxima à fisiológica.

DESCRITORES: Ravascularização miocárdica, pós-operatório. Estimulação cardíaca artificial. Marcapasso artificial.

Trabalho realizado no Hospital São Lucas da PUCRS - Porto Alegre, RS, Brasil.

Recebido para publicação em fevereiro de 2001.

* Do Hospital São Lucas da PUCRS - Porto Alegre, RS.

** Do Instituto de Cardiologia de Porto Alegre, RS.

*** Do Hospital de Clínicas de Porto Alegre, RS.

Endereço para Correspondência: Luciano Albuquerque - Av. Ipiranga, 6690 conj. 615. Porto Alegre - RS, Brasil. CEP: 90610-000.

e-mail:alb.23@terra.com.br 
Albuquerque L C, Sant'anna J R, Zago A J, Velho F J P, Petracco J B - Estudo comparativo das estimulações ventricular direita e biventricular no pós-operatório de revascularização miocárdica. Rev Bras Cir Cardiovasc 2002; 17(1): 61-72.

\section{INTRODUÇÃO}

O primeiro estudo procurando mostrar os benefícios da estimulação cardíaca artificial, na miocardiopatia dilatada refratária ao tratamento clínico, foi desenvolvido por HOCHLEITNER et al.(1). Analisando 16 pacientes com fração de ejeção média de $16 \%$, submetidos a marcapasso bicameral com retardo atrioventricular (AV) de $100 \mathrm{~ms}$, demostraram aumento de $20 \%$ na pressão arterial sistêmica, de $50 \%$ na $\mathrm{FE}$, e redução de $8 \%$ no índice cardiotorácico $(p<0,01)$. Em estudo de coorte realizado 5 anos mais tarde, este benefício hemodinâmico se mantinha ${ }^{(2)}$. Entretanto, nenhuma melhora dos parâmetros hemodinâmicos com estimulação AV foi encontrada nas séries de INNES et al.(3), LINDE et al. (4) e SHINBANE et al. (5).

O ensaio clínico cruzado desenvolvido por GOLD et al. ${ }^{(6)}$, que estudaram 12 pacientes com insuficiência cardíaca terminal, foi altamente contributivo no esclarecimento dessa questão. Os autores utilizaram marcapasso AV com retardo de $100 \mathrm{~ms}$, e aferiram invasivamente débito cardíaco (DC), FE, pressão arterial pulmonar e pressão arterial sistêmica. Em nenhuma destas variáveis houve benefício com a estimulação $\mathrm{AV}$, comparada ao ritmo sinusal ou à EVD isolada.

Frente aos conflitantes resultados dos marcapassos AV em pacientes com disfunção grave de VE, hipóteses baseadas no conceito da ressincronização ventricular passaram a ser especuladas. Desde que a maioria dos casos de miocardiopatia dilatada apresentam, além de disfunção contrátil, alterações na condução intraventricular que produzem alargamento do complexo QRS, a estimulação dos ventrículos em mais de um ponto, o mais sincronicamente possível, poderia produzir um complexo QRS mais estreito, portanto mais próximo do fisiológico, de forma a gerar um sinergismo mecânico mais efetivo, nas fibras miocárdicas. Isto poderia resultar em melhora da contratilidade miocárdica e do enchimento diastólico ventricular, com conseqüente benefício hemodinâmico.

Baseados em um caso exitoso apresentado por BAKKER et al. ${ }^{(7)}$, CAZEAU et al. (8), analisaram as alterações produzidas pela estimulação biventricular (EBV), em 8 pacientes com insuficiência cardíaca congestiva (ICC) e complexo QRS alargado (>120 $\mathrm{ms}$ ). Implantando eletrodos endocárdicos em átrio direito, ventrículo direito (ápice e septo alto) e ventrículo esquerdo (parede lateral), observaram aumento de $25 \%$ no índice cardíaco (IC) e redução de $17 \%$ na pressão capilar pulmonar $(p<0,01)$, em relação ao ritmo sinusal, efeito este que se manteve no seguimento médio de 6 meses.

Outros autores (9-12) também evidenciaram melhora hemodinâmica imediata na sincronização biventricular, em vários trabalhos com miocardiopatia dilatada e/ou ICC.
Com base nessa animadora experiência clínica inicial, foi desenvolvido um estudo multicêntrico denominado Medtronic Inc. Insync Study, com o objetivo de avaliar segurança e eficácia do sistema de estimulação biventricular, implantado no endocárdio através das veias cardíacas ${ }^{(13)}$. Em 68 de 81 pacientes elegíveis para a pesquisa $(84 \%)$, o implante de eletrodos pelo seio coronário foi exitoso, havendo, após o período de 10 meses, significativo encurtamento do complexo QRS, aumento do tempo de enchimento diastólico, e substancial melhora da qualidade de vida.

Apesar dessas evidências, AURICCHIO et al. ${ }^{(14)}$, em recente trabalho de revisão alertam para as dificuldades técnicas e riscos envolvidos no implante de eletrodos pelo seio venoso, ressaltando que limitações como variação anatômica das veias coronárias, e imprecisão a respeito do melhor local na parede ventricular, para otimizar o ganho hemodinâmico, poderiam tornar esta técnica pouco reprodutível.

Nesse sentido, PACHÓN MATEOS et al. (15) propuseram um modelo de estudo utilizando 2 eletrodos endocárdicos no VD, um no ápice e outro na porção alta do septo interventricular, implantados por via intravenosa convencional. Nos 5 pacientes inicialmente analisados, houve expressiva melhora do DC, da FE e da regurgitação mitral, associada à significativa diminuição da área do átrio esquerdo, e encurtamento do complexo QRS $(p<0,01)$, em relação à estimulação convencional do VD. Os autores concluíram que, em pacientes com grave ICC, esta estimulação ventricular, referida como bifocal, produz benefício similar à estimulação biventricular, configurando uma técnica mais simples e exeqüível do que as até então descritas.

Atualmente, existem quatro grandes ensaios clínicos internacionais em andamento, testando o real benefício hemodinâmico da estimulação biventricular na ICC grave.

O VIGOR CHF trial (16) está desenhado para uma análise seqüencial da estimulação biventricular, buscando separar a eventual influência do efeito placebo. Pacientes com ICC sintomática, complexo QRS igual ou superior a $120 \mathrm{~ms}$, $\mathrm{FE}$ abaixo de $30 \%$ e sem indicações convencionais ao uso de marcapasso, recebem implante de dois eletrodos endocárdicos, atrial e ventricular direitos, e de um eletrodo epicárdico no ventrículo esquerdo, e são randomizados em dois grupos: sem intervenção e com estimulação biventricular. Após 6 semanas, todos os pacientes recebem estimulação biventricular, até 18 semanas de seguimento. Os seguintes desfechos serão analisados na $6^{\circ}, 12^{\circ}$ e $18^{\circ}$ semanas: extração de oxigênio $\left(\mathrm{O}_{2}\right)$ em exercício máximo, qualidade de vida e variáveis ecocardiográficas de desempenho hemodinâmico, sendo os pacientes seus próprios controles. 
Albuquerque L C, Sant'anna J R, Zago A J, Velho F J P, Petracco J B - Estudo comparativo das estimulações ventricular direita e biventricular no pós-operatório de revascularização miocárdica. Rev Bras Cir Cardiovasc 2002; 17(1): 61-72.

O VENTAK CHF trial (16) constitui um ensaio clínico cruzado, desenhado para testar o efeito da estimulação biventricular em casos de insuficiência cardíaca grave, nos quais também exista indicação de cardioversor desfibrilador (CD) implantável. Pacientes com FE menor que 35\%, complexo QRS igual ou superior a $120 \mathrm{~ms}$, e submetidos a implante de CD com recurso de estimulação multicameral, após um mês são randomizados para receberem estimulação biventricular ou manterem o marcapasso desligado. Após 3 meses, os pacientes são cruzados, e acompanhados por mais 3 meses. Serão analisados no $3^{\circ}$ e $6^{\circ}$ meses os seguintes desfechos: extração de $\mathrm{O}_{2}$ em exercício máximo, qualidade de vida, segurança do sistema $C D$, e variáveis ecocardiográficas de desempenho hemodinâmico. À semelhança do estudo VIGOR CHF, os pacientes serão seus próprios controles.

O PATH-CHF study (17) testa a hipótese de que, em casos de ICC moderada a grave, as estimulações ventricular esquerda e biventricular podem determinar melhora clínica e de variáveis hemodinâmicas, a curto e longo prazos. Através do implante de eletrodos atrial e ventricular direitos (via endocárdica), e de eletrodo ventricular esquerdo (via epicárdica), os pacientes receberão estimulação univentricular ou biventricular por 4 semanas; após, permanecerão sem marcapasso também por 4 semanas, para testar o efeito placebo; e, por fim, os grupos terão invertidas as formas de estimulação, sendo observados por mais 4 semanas. O estudo pretende arrolar 53 casos de ICC, em 7 centros da Europa, e acompanhá-los por até 3 anos.

O MUSTIC study, cujo delineamento e dados preliminares foram apresentados no $21^{\circ}$ Congresso da North American Society of Cardiac Pacing and Electrophysiology (NASPE), constitui um ensaio clínico cruzado, em execução em 17 centros europeus, que testa eficácia e segurança da estimulação biventricular em casos de ICC grave. O estudo envolve dois grupos de pacientes, o primeiro com ritmo sinusal e sem indicação convencional ao uso de marcapasso, e o segundo com fibrilação atrial e ablação do nódulo AV: o grupo 1 é randomizado a receber estimulação biventricular ou permanecer com - marcapasso desligado, e o grupo 2 a receber estimulação ventricular direita convencional ou biventricular. Os principais desfechos analisados são tolerância ao exercício, extração máxima de $\mathrm{O}_{2}$ durante o esforço, qualidade de vida, e necessidade de hospitalização, sendo os subgrupos cruzados após 12 semanas. Os resultados preliminares de 63 pacientes demonstram que a EBV determinou, em ambos os grupos, aumento de $23 \%$ na tolerância ao exercício, de $8 \%$ na extração de $\mathrm{O}_{2}$, de $30 \%$ no escore de qualidade de vida, e ocorrência 3 vezes menor de hospitalizações, em comparação à EVD.

Estes quatro ensaios clínicos representam os maiores estudos empregando marcapassos no tra- tamento da disfunção sistólica grave de VE, com resultados finais aguardados em curto prazo.

Por outro lado, a cardiopatia isquêmica representa a causa mais comum de óbito nos países desenvolvidos, devendo crescer futuramente em torno de $40 \%$, quanto à prevalência e mortalidade, devido ao aumento da expectativa geral de vida da população (18).

Desde a sua criação, a cirurgia de revascularização miocárdica (CRM) popularizou-se enormemente como instrumento potencialmente capaz de modificar a história natural dessa doença, a ponto de tornar-se, nos Estados Unidos e no Canadá, a mais comum operação de grande porte realizada entre os anos 70 e 90 (19).

O implante intra-operatório de eletrodo(s) epimiocárdico(s) temporário(s) faz parte da rotina da CRM, e tem o objetivo de poder melhorar a performance hemodinâmica de pacientes que desenvolvem baixo débito cardíaco e/ou bloqueio $\mathrm{AV}$, após a saída de circulação extracorpórea, ou no pós-operatório recente.

Não obstante ser a estimulação artificial do VD o método mais empregado e difundido nessa situação, HARTZLER et al.(20), estudando uma série de 10 pacientes submetidos à operação cardíaca, relataram maior benefício hemodinâmico através da estimulação AV seqüencial, com intervalo de condução AV menor que o intrínseco. Entretanto, somente 4 dos casos estudados apresentavam disfunção ventricular, e apenas um foi submetido à CRM.

Para determinar o efeito do marcapasso temporário $\mathrm{AV}$ em pacientes coronariopatas, BETOCCHI et al.(21) analisaram 13 pacientes com cardiopatia isquêmica estabilizada e função ventricular normal. Contrariamente aos achados de Hartzler, a estimulação AV seqüencial determinou significativa piora do índice cardíaco e do enchimento ventricular, quando comparada à estimulação atrial.

FOSTER et al. (22), por sua vez, testaram um modelo de marcapasso átrio-biventricular em 18 pacientes submetidos à CRM, evidenciando significativa melhora do IC e da resistência vascular sistêmica, quando comparado à estimulação AV seqüencial direita e esquerda $(p<0,05)$. Entretanto, como $80 \%$ dos pacientes apresentavam função sistólica do VE normal, o pequeno embora estatisticamente provado benefício hemodinâmico (10\%) foi considerado insignificante do ponto de vista clínico. Em sua conclusão, os próprios autores ressaltavam a necessidade de estudos futuros, para identificar subgrupos de pacientes potencialmente mais favorecidos, como os portadores de disfunção ventricular.

Até o momento, não existem trabalhos que procurem reproduzir o benefício sugerido da estimulação biventricular na miocardiopatia dilata- 
Albuquerque L C, Sant'anna J R, Zago A J, Velho F J P, Petracco J B - Estudo comparativo das estimulações ventricular direita e biventricular no pós-operatório de revascularização miocárdica. Rev Bras Cir Cardiovasc 2002; 17(1): 61-72.

da, em pacientes com comprometimento da função sistólica do VE, no pós-operatório precoce de CRM.

Tal evidência poderia ampliar o arsenal terapêutico até então oferecido a pacientes que apresentam bloqueio atrioventricular e/ou baixo débito cardíaco pós-operatório, contribuindo para sua otimização hemodinâmica.

O objetivo do presente estudo é avaliar o efeito hemodinâmico imediato da estimulação artificial biventricular, no pós-operatório de operação de revascularização miocárdica, em pacientes com disfunção sistólica do ventrículo esquerdo, comparando-a à estimulação univentricular direita.

\section{CASUÍSTICA E MÉTODOS}

Foram estudados 13 pacientes com cardiopatia isquêmica e comprometimento da função ventricular esquerda, submetidos à operação de revascularização miocárdica, no Hospital São Lucas da Pontifícia Universidade Católica do Rio Grande do Sul, no período entre 10 de outubro de 1999 e 17 de maio de 2000 , e designados para a pesquisa conforme os critérios descritos a seguir:

\section{Critérios de Inclusão}

- Pacientes com doença coronária multiarterial, e indicação de operação de revascularização com circulação extracorpórea (CEC).

- Fração de ejeção do ventrículo esquerdo inferior a $50 \%$, estimada pela ventriculografia, obtida em estudo hemodinâmico.

\section{Critérios de Exclusão}

- Infarto do miocárdio em período pré-operatório de até 30 dias, e/ou IAM intra-operatório, ambos definidos por critérios eletrocardiográficos e enzimáticos.

- Revascularização incompleta do miocárdio, definida como o implante de, no mínimo, um enxerto a menos do que o planejado no pré-operatório.

- Ocorrência de arritmias cardíacas, no período intra ou pós-operatório.

- Uso de drogas antiarrítmicas, arritmogênicas e/ou inotrópicas, no momento da aferição das variáveis.

- Restrição clínica à realização de ecocardiograma transtorácico no $5^{\circ}$ dia pós-operatório.

- Janela ecocardiográfica inadequada para a análise das variáveis.

- Discordância do paciente em participar do estudo.
As variáveis em estudo foram a duração do complexo QRS (DUR/QRS), aferida por eletrocardiograma convencional de 12 derivações, o diâmetro do átrio $E$ (DAE), os diâmetros sistólico e diastólico finais do VE (DSVE e DDVE), a fração de encurtamento percentual do VE (delta D) e a fração de ejeção do VE (FEVE), analisados por ecocardiograma transtorácico em modo $\mathrm{M}$, com cortes orientados pelo exame bidimensional.

Os eletrodos epimiocárdicos foram implantados durante a fase de aquecimento, em circulação extracorpórea, após a realização das anastomoses proximais. O eletrodo ventricular direito foi posicionado na face anterior da via de saída, próximo ao sulco interventricular, enquanto o eletrodo ventricular esquerdo localizou-se na porção média da face póstero-lateral, após o ramo marginal obtuso da artéria circunflexa. Em ambas as situações, o fio de marcapasso foi implantado penetrando superficialmente a parede ventricular, de forma a que todo o segmento exposto ("desencapado") do eletrodo permanecesse totalmente sepultado no miocárdio, para evitar-se o risco de perda de comando, ou falha de sensibilidade ${ }^{(23)}$. Após o implante, o sistema de estimulação foi testado por um gerador bicameral de pulso, para assegurar-se sua confiabilidade. O mesmo cirurgião realizou as operações de revascularização, e o implante dos eletrodos epimiocárdicos, em todos os casos estudados.

A estimulação artificial e a aferição das variáveis em estudo foram realizadas no $5^{-0}$ dia pósoperatório, com os pacientes em condições clínicas estáveis, extubados e sem o uso de drogas inotrópicas ou de dispositivos de assistência circulatória, no Laboratório de Ecocardiografia do Hospital São Lucas da PUCRS. Todas as variáveis em estudo foram analisadas em três diferentes momentos, no mesmo paciente: inicialmente, em ritmo sinusal; a seguir, ativou-se o modo de estimulação ventricular direito, e aguardou-se o tempo de 5 minutos para adequação hemodinâmica, procedendo-se a nova coleta dos dados (grupo EVD); por fim, utilizou-se o modo de estimulação biventricular, igualmente aguardouse o tempo de 5 minutos, e realizou-se novamente as mensurações (grupo EBV). Os pacientes mantiveram-se em repouso, na posição de decúbito dorsal (medidas eletrocardiográficas) e decúbito lateral esquerdo (medidas ecocardiográficas), sob as mesmas condições de freqüência cardíaca, freqüência respiratória, retorno venoso, atividade simpática e climatização do ambiente.

Foram utilizados dois eletrodos epimiocárdicos bipolares Biotronik $\AA$, modelo MHW 150-BP, exteriorizados em cada lado do hemitórax correspondente ao ventrículo estimulado. O mesmo gerador bicameral de pulso, Biotronik ${ }^{\circledR}$ modelo EDP 
Albuquerque L C, Sant'anna J R, Zago A J, Velho F J P, Petracco J B - Estudo comparativo das estimulações ventricular direita e biventricular no pós-operatório de revascularização miocárdica. Rev Bras Cir Cardiovasc 2002; 17(1): 61-72.

30/A, foi empregado em todos os casos; de acordo com a classificação da North American Society of Pacing and Electrophysiology ${ }^{(24)}$, selecionaramse os seguintes modos de geração de pulso: VVI para estimulação ventricular direita, e "DDD" para o modo VVI biventricular, empregando-se, neste último, o intervalo de condução intercameral mínimo do aparelho, de $10 \mathrm{~ms}$. O eletrodo VD foi conectado à entrada correspondente à câmara atrial, enquanto o eletrodo VE à entrada ventricular do gerador. Em todos casos, o gerador foi ajustado com amplitude de pulso de 3 volts, com duração de estímulo de $0,5 \mathrm{~ms}$, e em freqüência de 10 bpm superior à sinusal.

Para a análise dos resultados, foi utilizado o programa SPSS versão 6.0, para Windows. Os resultados das variáveis em estudo foram submetidos ao teste de t de Student para amostras pareadas, para comparação entre os dois grupos intervidos. Em todas as análises, considerou-se significativo o valor de $p<0,05$.

O projeto deste estudo foi analisado e aprovado pelo Comitê de Ética em Pesquisa, e pela Comissão Científica, da Faculdade de Medicina da Pontifícia Universidade Católica do Rio Grande do Sul. Todos os pacientes foram esclarecidos pelo pesquisador, sobre objetivos e riscos do estudo; e, concordando em participar, assinaram Termo de Consentimento Informado.

\section{RESULTADOS}

De quinze pacientes inicialmente elegíveis para o protocolo do estudo, dois foram excluídos: um por infarto do miocárdio intra-operatório e um por janela ecocardiográfica inadequada, devida à obesidade mórbida. Efetivamente, foram estudados 13 casos, dos quais $10(77 \%)$ eram do sexo masculino e $3(23 \%)$ do sexo feminino. A idade variou de 48 a 73 anos, com uma idade média de 60,5 anos. De acordo com a manifestação clínica predominante, se angina ou insuficiência cardíaca, e segundo os critérios da NYHA, 2 (15\%) pacientes encontravam-se em classe funcional II, $7(54 \%)$ em classe funcional III, e 4 (31\%) em classe funcional IV (Gráfico 1).

A média da fração de ejeção pré-operatória, estimada pelo cateterismo cardíaco, foi de $34 \%$ e não diferiu significativamente da fração de ejeção pós-operatória, calculada pelo ecocardiograma, em ritmo sinusal, de $36 \%(p=0,88)$.

O número médio de enxertos realizados foi de 2,9 ; em $85 \%$ das operações $(n=11)$, a artéria torácica interna esquerda foi utilizada para revascularização da artéria descendente anterior. O tempo médio do procedimento cirúrgico foi de 168 minutos (dp 25), o tempo médio de CEC 60 minutos (dp 11), e o tempo médio de isquemia
GRÁFICO 1

CLASSE CLÍNICA, SEGUNDO OS CRITÉRIOS DA NEW YORK HEART ASSOCIATION

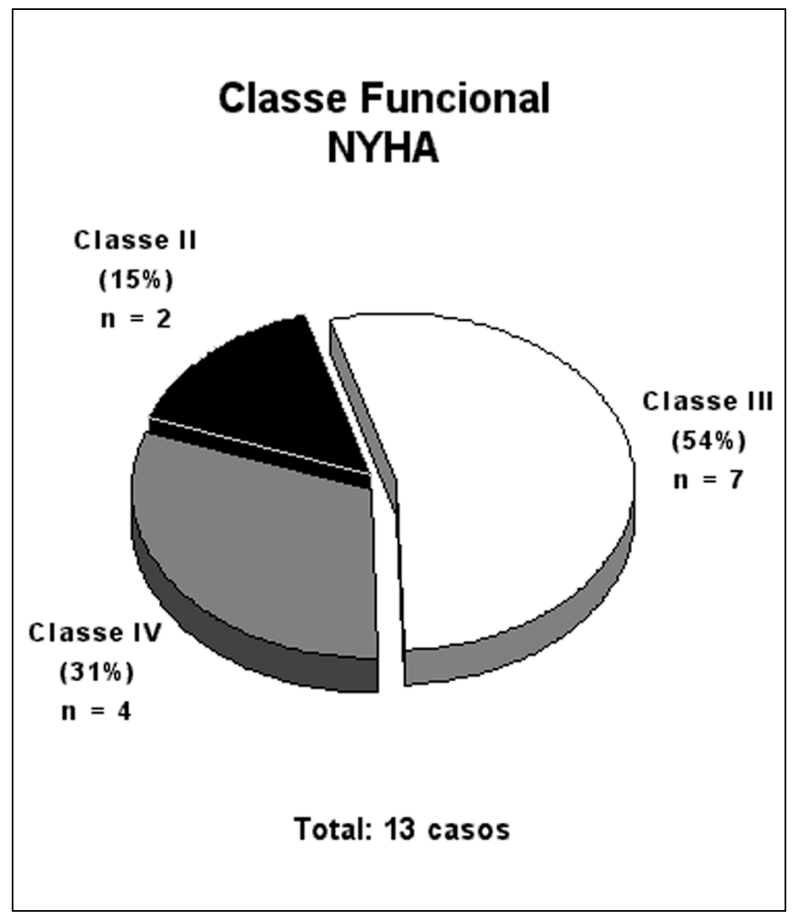

miocárdica 33 minutos. O uso de balão intra-aórtico foi necessário em 9 casos $(70 \%)$, para otimização hemodinâmica peri-operatória.

O eletrocardiograma em ritmo sinusal demonstrou infarto prévio do miocárdio em 10 (70\%) casos; em relação à presença de distúrbios de condução, $6(46 \%)$ exames foram considerados normais, 4 (31\%) apresentaram retardo na condução intraventricular, 2 (15\%) preencheram critérios de bloqueio de ramo esquerdo e 1 (8\%) de bloqueio de ramo direito (Gráfico 2 ).

A análise ecocardiográfica evidenciou alterações segmentares significativas da contratilidade do VE (hipocinesia ou acinesia) em $9(70 \%)$ pacientes, mobilidade anômala do septo interventricular em 7 (54\%) e insuficiência mitral de grau leve em 3 (23\%) casos.

Não houve dificuldade técnica no implante dos eletrodos epimiocárdicos e, em nenhum caso, foi necessária a ativação do sistema de marcapasso, por eventual dificuldade de adequação hemodinâmica após descontinuada a CEC. Não foram observados efeitos colaterais e/ou desconforto, durante as fases de estimulação artificial e aferição das variáveis, e não houve óbito hospitalar.

Em ritmo sinusal, a DUR/QRS média foi de 104,6 ms (dp 16,7); quando em estimulação ventricular direita, a média foi de 184,9 ms (dp 25,9), e de 126,2 ms (dp 36,9) com a estimulação biventricular $(p<0,001)$ (Gráfico 3). 
O DAE médio em ritmo sinusal foi de $40,1 \mathrm{~mm}$ (dp 4,0); durante estimulação ventricular direita, a média também foi de $40,1 \mathrm{~mm}$ (dp 3,7), enquanto a média de $35,4 \mathrm{~mm}$ (dp 3,9) foi encontrada em estimulação biventricular $(p<0,001)$. A média do DSVE em ritmo sinusal foi de $48 \mathrm{~mm}$ (dp 12,1), sob estimulação ventricular direita foi de $49,2 \mathrm{~mm}$ (dp $12,6)$, e de $42,2 \mathrm{~mm}$ (dp 11,9) durante estimulação biventricular $(p<0,001)$. A média do DDVE em ritmo sinusal foi de $58,6 \mathrm{~mm}$ (dp 10,4); em estimulação ventricular direita, a média foi de $59,1 \mathrm{~mm}$ (dp $11,2)$, e de $51,8 \mathrm{~mm}(\mathrm{dp} \mathrm{9,8)}$ durante estimulação biventricular $(p<0,001)$ (Gráfico 4).

A média da delta $D$ em ritmo sinusal foi de $18 \%$ (dp 7), em estimulação ventricular direita igualmente $18 \%$ (dp 7), e foi de 22\% (dp 8) durante estimulação biventricular $(p=0,017)$. A média da FEVE foi de $36 \%$ (dp 13) em ritmo sinusal, de $33 \%$ (dp 14) em estimulação ventricular direita, e de 46\% (dp 17) com a estimulação biventricular $(p<0,001)$. (Gráfico 5$)$.

No Gráfico 6, uma representação gráfica ilustra a magnitude das diferenças percentuais observadas, em cada desfecho estudado. Se considerarmos a estimulação ventricular direita convencional como referência (ponto zero), podemos verificar que, durante a estimulação biventricular, houve uma redução de $32 \%$ na DUR/QRS, o DAE médio diminuiu $12 \%$, o DSVE médio foi reduzido em $14 \%$, houve redução de $13 \%$ no DDVE médio, a média da delta $D$ aumentou $27 \%$ e ocorreu um aumento de $38 \%$ na FEVE média.
GRÁFICO 2

ACHADOS ELETROCARDIOGRÁFICOS PRÉOPERATÓRIOS EM RITMO SINUSAL

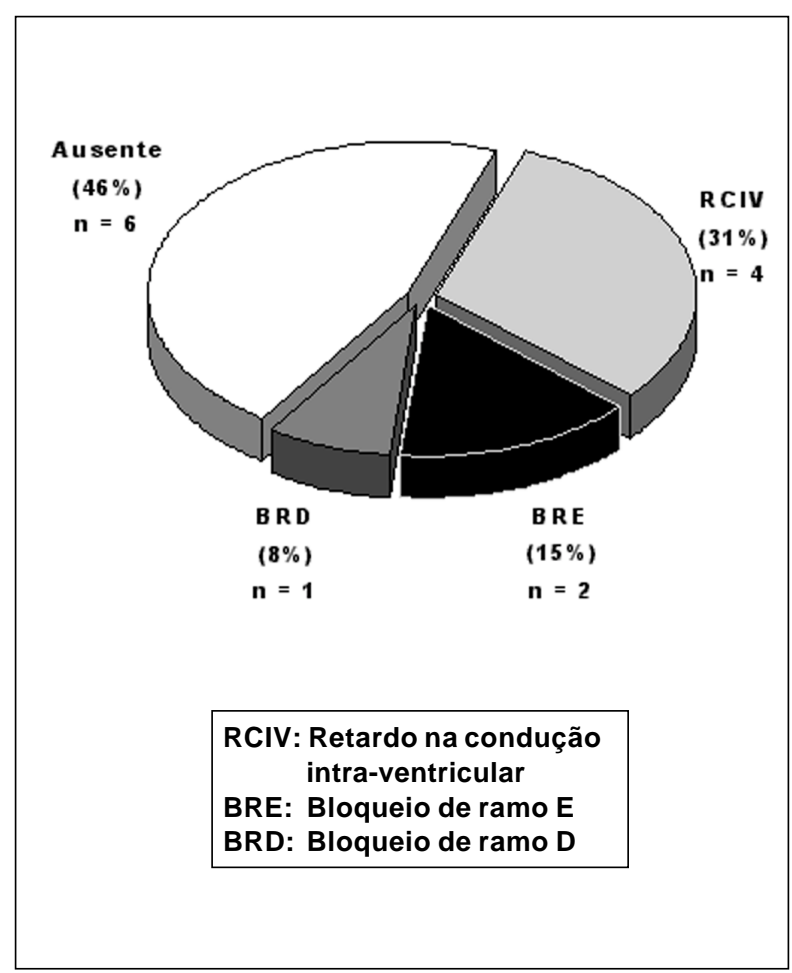

\section{GRÁFICO 3}

DURAÇÃO MÉDIA DO COMPLEXO QRS (DUR/QRS)

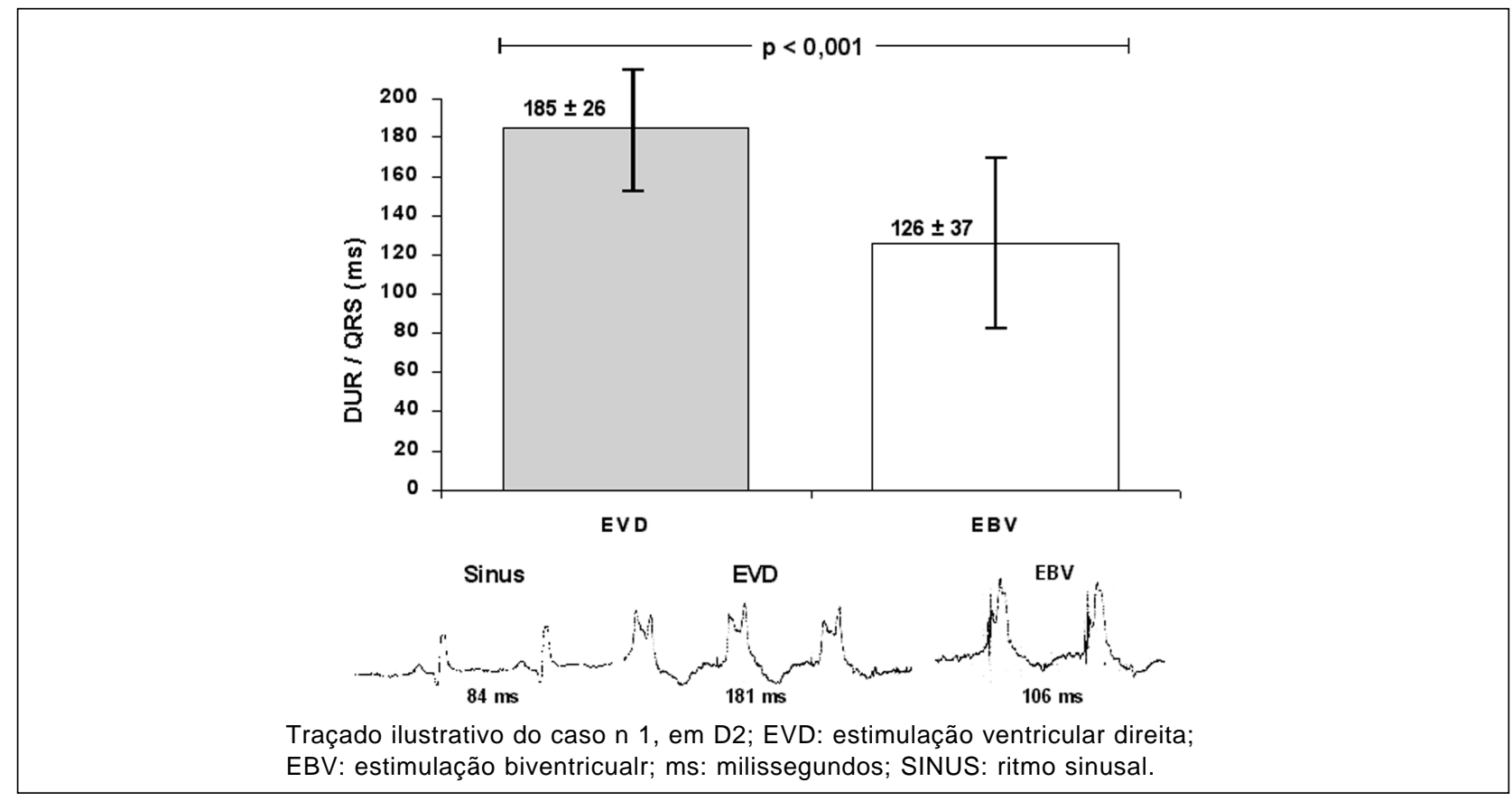




\section{GRÁFICO 4}

\section{DIÂMETRO INTRA-CAMERAIS MÉDIOS, EM MODO M DO ECOCARDIOGRAMA}

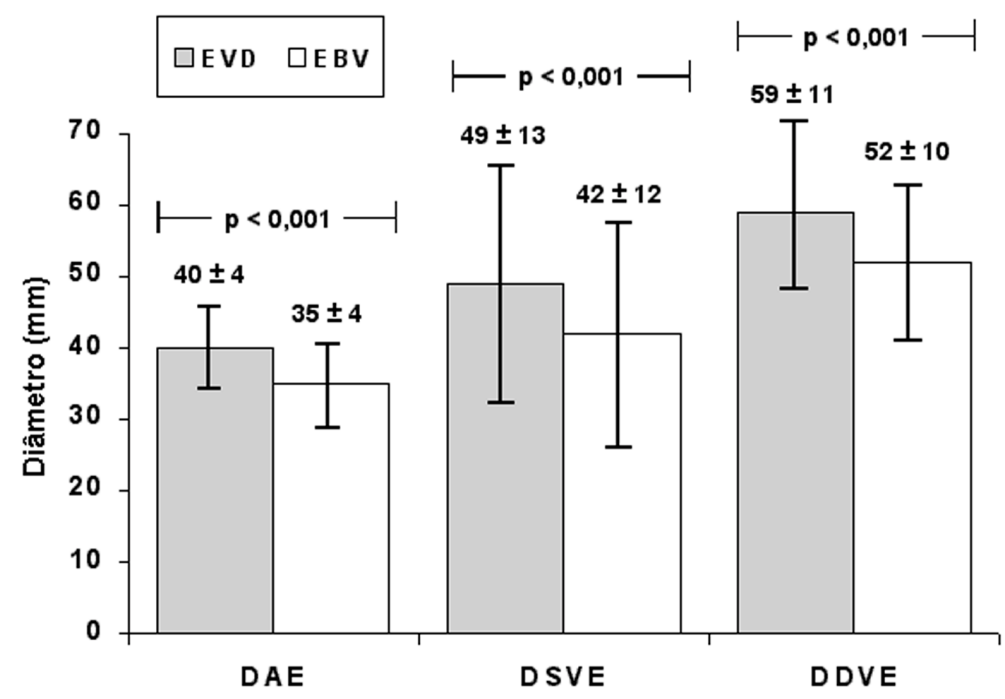

DAE: diâmetro do átrio esquerdo; DSVE: diâmetro sistólico final do VE; DDVE: diâmetro diastólico final do VE; EVD: estimulação ventricular direita; EBV: estimulação biventricular; ms: milissegundos.

GRÁFICO 5

FRAÇÕES MÉDIAS DO VENTRÍCULO ESQUERDO

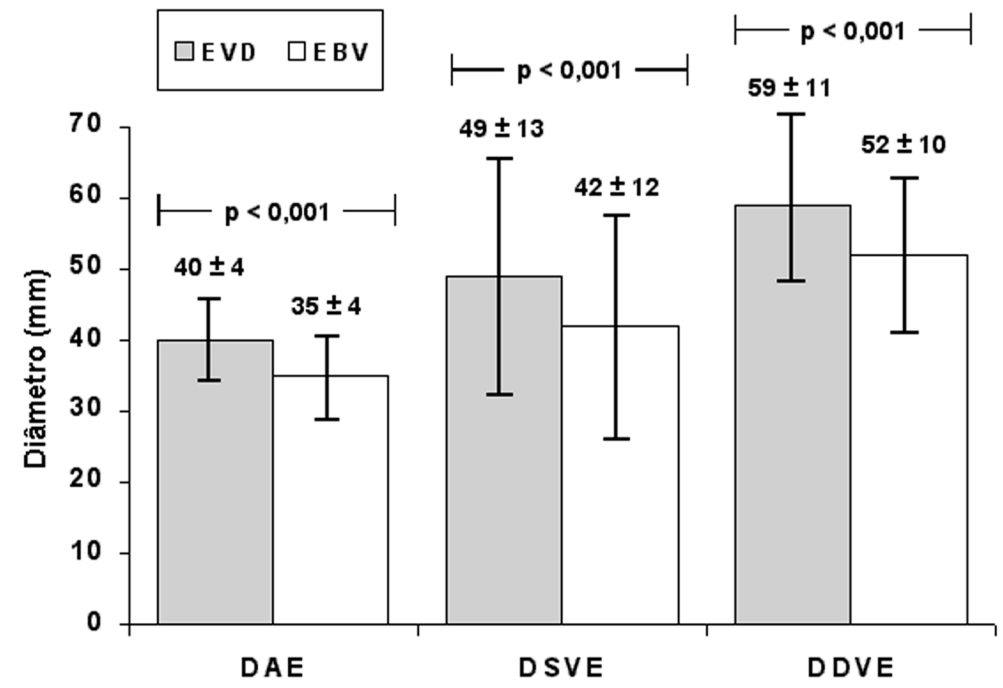

Delta D: fração de encurtamento; FEVE: fração de ejeção; EVD: estimulação ventricular direita; EBV: estimulação biventricular. 
Albuquerque L C, Sant'anna J R, Zago A J, Velho F J P, Petracco J B - Estudo comparativo das estimulações ventricular direita e biventricular no pós-operatório de revascularização miocárdica. Rev Bras Cir Cardiovasc 2002; 17(1): 61-72.

\section{GRÁFICO 6}

Magnitude das diferenças porcentuais observados com a ebv

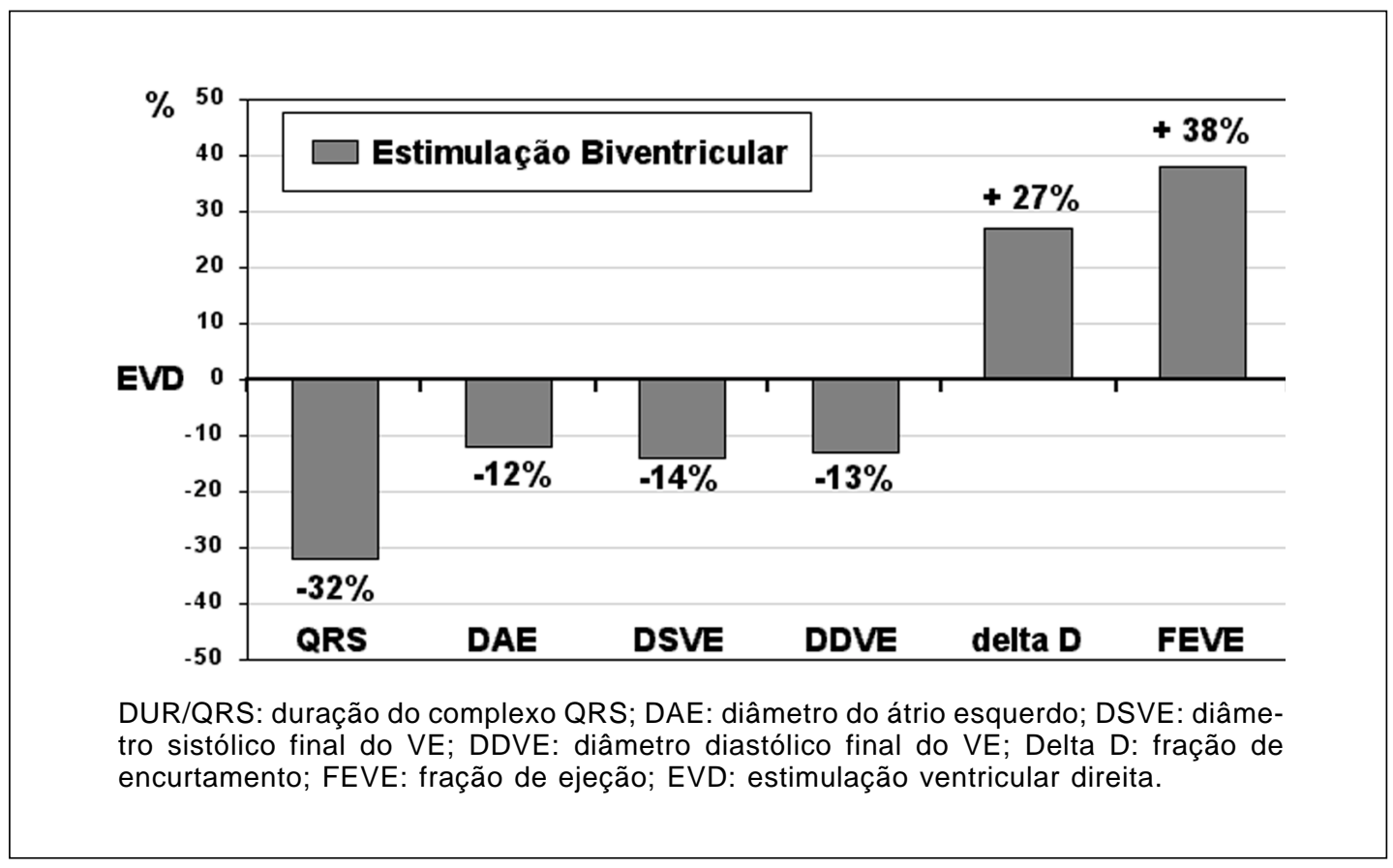

\section{COMENTÁRIOS}

O estado atual da estimulação cardíaca artificial propõe que, além de corrigir anormalidades de ritmo e condução, os sistemas de marcapasso busquem uma sincronização máxima das câmaras cardíacas, para que se obtenha a melhor performance hemodinâmica. Em pacientes com disfunção ventricular, nos quais a propagação intraventricular do estímulo é freqüentemente mais lenta e a dissincronia ventricular mais deletéria, torna-se fundamental, para a manutenção de um débito cardíaco adequado, que a ativação eletromecânica dos ventrículos ocorra de forma homogênea.

A tentativa de melhorar o estado hemodinâmico de pacientes com insuficiência cardíaca grave através da estimulação $A V$ com intervalo curto, não obstante os resultados iniciais positivos ${ }^{(1,2)}$, demonstrou-se inefetiva em trabalhos mais consistentes, que constataram que o marcapasso AV produzia uma ativação eletromecânica inversa dos ventrículos $^{(3-6)}$. Com o mesmo propósito, o conceito da estimulação biventricular simultânea tem se difundido como potencialmente benéfico em casos de grave disfunção miocárdica. Os resultados de séries utilizando estimulação biventricular (7-13) ou ventricular direita bifocal (15), no tratamento da miocardiopatia dilatada, motivou o delineamento de quatro grandes ensaios clínicos, ainda em andamento $(16,17)$.
Por outro lado, no tratamento da cardiopatia isquêmica, o avanço das técnicas percutâneas de revascularização tem progressivamente restringido os candidatos à CRM, a um grupo de pacientes mais graves, onde a disfunção sistólica de VE é marcadamente predominante. Nesses casos, em que a ocorrência de bradiarritmias e baixo débito cardíaco no pós-operatório não é infreqüente, é desejável o melhor desempenho cardiocirculatório.

O presente estudo foi desenhado com o intuito de averiguar se os resultados sugeridos com a estimulação biventricular na insuficiência cardíaca podem ser reproduzidos em pacientes coronariopatas com disfunção ventricular, no pós-operatório de CRM.

$\mathrm{Na}$ determinação da população a ser estudada, procuramos incluir pacientes candidatos à CRM, com doença multiarterial e disfunção sistólica de VE. Apenas a seleção de casos com complexo QRS anormalmente alargado, restringiria sobremaneira o grupo em estudo, diminuindo a validade externa da pesquisa. $O$ potencial viés de seleção, devido à utilização de uma estimativa hemodinâmica da FEVE como critério de inclusão, foi controlado pela demonstração de equivalência da mesma com a FEVE verificada no ecocardiograma pós-operatório, em ritmo sinusal $(p=0,88)$. A exclusão de pacientes com IAM recente, IAM intra-operatório, revascularização incompleta ou arritmias, justifica-se pelo risco de instabilidade elétrica, pela possibilidade de resposta 
Albuquerque L C, Sant'anna J R, Zago A J, Velho F J P, Petracco J B - Estudo comparativo das estimulações ventricular direita e biventricular no pós-operatório de revascularização miocárdica. Rev Bras Cir Cardiovasc 2002; 17(1): 61-72.

inadequada à estimulação artificial e pela eventual perda de comando do marcapasso. Foram ainda excluídos casos em que o uso de drogas antiarrítmicas, arritmogênicas ou inotrópicas pudesse confundir a leitura dos resultados.

O implante dos eletrodos epimiocárdicos foi procedido pelo mesmo cirurgião, para evitar eventuais diferenças de posicionamento. Embora não haja consenso entre os autores sobre o melhor ponto de contato na parede ventricular, nossa opção de implante na superfície parasseptal do VD, e na parede livre do VE, baseou-se em importantes estudos prévios. GRAAS et al. (13), em um ensaio clínico multicêntrico para testar a eficácia da EBV em 68 pacientes com ICC, evidenciaram que o melhor benefício hemodinâmico ocorreu quando o eletrodo ventricular direito foi posicionado na via de saída, em comparação ao ápice. HATALA et al.(25), por sua vez, através do mapeamento endocárdico de pacientes com infarto prévio em VE, verificaram que a região mais tardiamente ativada durante a EVD era a parede posterior do VE. Com base nesses achados, LECLERCQ et al.(10), em um ensaio clínico testando a EBV em 18 pacientes com ICC grave, escolheram locais para estimulação simultânea que correspondessem ao ponto de ativação mais precoce no VD (junto ao septo), e mais tardio no VE (face póstero-lateral); com este modelo, por nós reproduzido, os autores demonstraram aumento significativo do índice cardíaco, em comparação à estimulação atrial e do VD.

Arbitramos o $5^{\circ}$ dia de pós-operatório para testar os modos de estimulação, por entendermos que fatores como dor, presença de drenos torácicos ou catetéres intravenosos, internação em Unidade de Tratamento Intensivo, ou o uso de drogas vasoativas pudessem alterar o tônus simpático, de forma a confundir os resultados. Pela mesma razão, todos os pacientes foram avaliados em repouso, sob as mesmas condições de freqüência cardíaca e respiratória, retorno venoso, atividade simpática e climatização do ambiente.

Utilizamos a ecocardiografia transtorácica para avaliação das variáveis hemodinâmicas por ser um método não invasivo, de fácil execução, sem limitações para uso no $5^{\circ}$ dia de pós-operatório e, portanto, bastante exeqüível para o modelo proposto. Um aspecto questionável, no presente estudo, é termos utilizado medidas ecocardiográficas em um grupo de pacientes com alta incidência de alterações segmentares da contratilidade ventricular (70\%), o que poderia constituir um viés de aferição. Entretanto, o fato de os pacientes terem sido seus próprios controles, e as medidas terem sido feitas no mesmo ponto de corte, pelo mesmo observador, controlou de forma efetiva este potencial viés.
Alterações da propagação intraventricular do estímulo elétrico são freqüentes em pacientes com depressão da função miocárdica( ${ }^{(26)}$, e nossos achados não contrariam este conceito. Em $54 \%$ dos casos estudados, houve alterações eletrocardiográficas expressivas de retardo na condução intraventricular, sendo a duração média do complexo QRS de 105 ms. Com a estimulação VD, observou-se alargamento do QRS (média $185 \mathrm{~ms}$ ), o qual não foi clinicamente expressivo, a ponto de determinar piora das variáveis hemodinâmicas. Com o marcapasso BV, o QRS médio resultante foi de 126 ms, significativamente mais estreito que o observado no grupo VD $(p<0,001)$, e com duração próxima ao grupo sinusal. De acordo com o relato de ALONSO et al. (12), na estimulação BV, a melhora dos parâmetros hemodinâmicos correlaciona-se linearmente com o estreitamento do QRS. Os autores estudaram 26 portadores de ICC refratária e complexo QRS alargado, submetidos ao implante endocárdico de marcapasso biventricular. Durante a estimulação artificial, foram analisadas a FEVE, a tolerância ao exercício, a classe funcional e a duração do complexo QRS. A análise dos resultados evidenciou que, nos casos que apresentaram melhora hemodinâmica, o complexo QRS produzido pela EBV era significativamente mais estreito do que o presente nos pacientes não-responsivos, o que observamos claramente em nosso estudo, na comparação entre os grupos EVD e EBV.

GRINES et al. (27) compararam variáveis ecocardiográficas de 18 pacientes com retardo da condução intraventricular e sem outras doenças cardíacas, às de 10 indivíduos normais. No grupo em estudo, a FE regional era significativamente diminuída ao nível do septo interventricular (40\% vs $67 \%$ - $p<0,001)$, sem que houvesse diferenças na FE apical e lateral, entre os grupos. A perda da contribuição da mobilidade septal resultou em redução da FE global dos pacientes com condução anormal, em relação aos controles (54\% vs $62 \%$ $\mathrm{p}<0,005)$. Nesse trabalho, a magnitude da mobilidade sistólica septal e a FE regional ao nível do septo tiveram forte correlação negativa com a dissincronia interventricular $(r=-0,86$ e $-0,85)$.

Uma vez que o movimento paradoxal do septo interventricular é freqüente no $\mathrm{PO}$ de revascularização miocárdica, e que mais da metade de nossos casos $(n=7)$ apresentavam este achado, é possível que a estimulação BV, deflagrando uma ativação sincrônica das câmaras ventriculares e do septo, tenha incrementado a contratilidade global, a partir do ganho regional a este nível. Embora tenhamos observado aumento do movimento septal anômalo durante a EVD e atenuação do mesmo no exame ecocardiográfico dos 7 pacientes, este achado foi fruto da interpretação subjetiva do examinador e, 
Albuquerque L C, Sant'anna J R, Zago A J, Velho F J P, Petracco J B - Estudo comparativo das estimulações ventricular direita e biventricular no pós-operatório de revascularização miocárdica. Rev Bras Cir Cardiovasc 2002; 17(1): 61-72.

portanto, não sujeito ao tratamento estatístico como as demais variáveis de interesse. Estudos futuros, analisando especificamente este subgrupo, poderão definir de forma mais consistente estas impressões.

No âmbito da aplicabilidade terapêutica, a análise crítica dos nossos resultados devem reportar-se a estudos semelhantes, já existentes. FOSTER et al. ${ }^{(22)}$, em um modelo de estimulação temporária aplicado em 18 pacientes com índice cardíaco normal, testaram o efeito hemodinâmico de marcapassos atrial, AV direito, AV esquerdo e atriobiventricular (ABV), com retardo AV fixo de $150 \mathrm{~ms}$, no pós-operatório imediato de CRM. Houve aumento significativo do IC e expressiva queda da resistência vascular sistêmica com a estimulação $A B V$, sem diferença dessas variáveis entre os outros 3 grupos. Este resultado, embora comprove a eficácia da ressincronização dos ventrículos, merece algumas considerações. Na medida em que $80 \%$ dos casos apresentavam FEVE superior a $40 \%$, e a média do IC era superior a 2,9 antes das intervenções, a pouca magnitude do ganho hemodinâmico (10\%) pode ter significância clínica discutível, no modelo proposto; o posicionamento do eletrodo do VE na região parasseptal pode ter determinado um resultado subótimo na estimulação ABV; a aferição das variáveis ocorreu entre 12 a 36 horas após a operação, onde o uso de drogas vasoativas é comum e pode ter influenciado nos achados; além disso, sabe-se que, em pacientes sob estimulação AV seqüencial, o ótimo intervalo de condução tende a ser variável, e deveria ser individualizado, caso a caso (28).

HARTZLER et al. (20) utilizaram retardo de condução AV individualizado e mais curto do que o intervalo intrínseco, em 10 pacientes submetidos à operação cardíaca, e compararam as alterações no débito cardíaco produzidas pelas estimulações AV seqüencial e ventricular direita convencional. A elevação média de $34 \%$ no DC com o marcapasso $A V$, em relação ao modo VVI, levou os autores a sugerirem esta forma de estimulação temporária, como preferencial no PO de operação cardíaca, a menos que houvesse taquiarritmias supraventriculares. Entretanto, no modelo proposto, somente um caso foi submetido à CRM, e a aferição das variáveis foi realizada nas primeiras 12 horas de $\mathrm{PO}$, onde não somente o eventual uso de drogas inotrópicas, mas também as alterações decorrentes da CEC, cardioplegia e reperfusão miocárdica, poderiam ter influenciado nos resultados.

Entretanto, outros autores (21,29), analisando especificamente portadores de cardiopatia isquêmica, demonstraram claramente que a estimulação AV artificial determina queda do índice cardíaco e da taxa de enchimento ventricular rápido (peak filling rate), em relação à condução $\mathrm{AV}$ intrínseca. mesmo em pacientes com função miocárdica preservada. No estudo de BETOCCHI et al. (21), 13 pacientes com doença coronária clinicamente estabilizada, e com condução atrioventricular e intraventricular normais, foram submetidos à estimulação atrial e AV seqüencial. Os autores demonstraram que a assincronia induzida pelo marcapasso $\mathrm{AV}$, simulando uma situação de bloqueio de ramo esquerdo, determinou queda no índice cardíaco às custas da diminuição da taxa de enchimento ventricular rápido e do volume diastólico final do VE.

Estes achados de literatura indicam que, em pacientes com disfunção ventricular esquerda, o marcapasso AV seqüencial, além da necessidade de individualização do intervalo $A V$, não elimina os efeitos hemodinâmicos deletérios da dissincronia ventricular. Apesar do modelo de estimulação proposto por FOSTER et al. (22) simular uma seqüência de ativação elétrica semelhante à fisiológica em repouso e manter o sinergismo contrátil dos ventrículos, a necessidade de individualização do intervalo AV ideal pode constituir um fator limitante à sua aplicação clínica rotineira.

O delineamento por nós desenvolvido permitiu concluir que, em pacientes com disfunção sistólica de VE submetidos à CRM, a ressincronização ventricular gerada pela EBV determinou melhora do estado hemodinâmico, em relação à EVD convencional. Infelizmente, a extensão desta conclusão a pacientes em ritmo sinusal não foi possível, devido à diferença de freqüência cardíaca (10 bpm superior ao ritmo sinusal nos grupos estimulados), e à ausência da manutenção do sincronismo atrioventricular nos modos de estimulação. Nesse aspecto, o estudo pode ter apresentado a limitação de desconsiderar a condução AV nos marcapassos testados, o que, possivelmente, levaria à subestimação do benefício hemodinâmico potencial. No entanto, entendemos que o fato de termos reproduzido o mesmo modo de estimulação nos dois grupos intervindos, conforme metodologia previamente definida, não compromete os objetivos a que esta pesquisa se propôs.

Estudos futuros, empregando a estimulação AAI em pacientes com condução AV intacta, como critério para grupo controle, talvez possam ampliar as atuais conclusões.

No entanto, a evidência de que a EBV supera a EVD convencional, em termos de benefício hemodinâmico, pode ter importante significância terapêutica, já que, no grupo de pacientes estudados, pequenos percentuais de ganho no desempenho hemodinâmico podem determinar expressiva melhora clínica, como, por exemplo, condições para retirada do balão intra-aórtico; ademais, a possibilidade do aumento da performance cardiocirculatória 
Albuquerque L C, Sant'anna J R, Zago A J, Velho F J P, Petracco J B - Estudo comparativo das estimulações ventricular direita e biventricular no pós-operatório de revascularização miocárdica. Rev Bras Cir Cardiovasc 2002; 17(1): 61-72.

com menor consumo de $\mathrm{O} 2$, do que o obtido com drogas simpaticomiméticasc ${ }^{(20)}$ adiciona vantagens teóricas à estimulação artificial biventricular.

O presente estudo apresenta um modelo de fácil reprodutibilidade e execução, enquadrado dentro de um procedimento de rotina na operação cardíaca, que não adiciona custos, e que, de acordo com os resultados obtidos, pode oferecer uma opção terapêutica adicional no sentido de melhorar a performance hemodinâmica de pacientes com disfunção ventricular que apresentem bloqueio atrioventricular e/ou baixo débito cardíaco, no pós-operatório recente de operação de revascularização miocárdica.

\section{CONCLUSÕES}

A análise dos resultados do presente ensaio clínico sugere que:

- A estimulação biventricular determinou significativa redução na duração do complexo QRS, em comparação ao observado durante estimulação ventricular direita.

- A duração do complexo QRS gerado pela estimulação biventricular foi próxima à fisiológica.

- A estimulação biventricular determinou significativa redução do diâmetro do átrio esquerdo, do diâmetro sistólico final do ventrículo esquerdo e do diâmetro diastólico final do ventrículo esquerdo, em relação à estimulação ventricular direita.

- A estimulação biventricular resultou em aumento significativo da fração de encurtamento percentual do ventrículo esquerdo e da fração de ejeção do ventrículo esquerdo, em comparação à estimulação ventricular direita.

- Em pacientes com disfunção sistólica de ventrículo esquerdo, submetidos à operação de revascularização miocárdica, a estimulação biventricular determina uma performance hemodinâmica meIhor do que a estimulação ventricular direita.

RBCCV 44205-573

Albuquerque L C, Sant'Anna J R, Zago A J, Velho F J P, Petracco J B - Comparative study of right ventricular and biventricular stimulations in post-operative of myocardial revascularization. Rev Bras Cir Cardiovasc 2002; 17(1): 61-72.

ABSTRACT: Objective: In recent years, the ventricular resynchronization has been proposed as an assisting therapy in congestive heart failure. This study objective is to compare the electrocardiographic changing and the acute hemodynamics effects of right ventricular (RVS) and biventricular stimulation (BVS), in post-operative of myocardial revascularization with cardiopulmonary bypass.

Material and Methods: In a cross-over trial, thirteen patients with multiarterial coronary disease and ejection fraction lower than $50 \%$, were submitted to right ventricular and biventricular epicardial temporary stimulation, in 5th post-operative day. The variables researched were lenght of time of the QRS complex, diameter of left atrium (LA) and left ventricle (LV), the LV shortening fraction and LV ejection fraction. The results from two groups were compared through the Student's $t$ test for paired observations and the value $p<0.05$ was considered significant.

Results: The duration of QRS complex was $185 \pm 26 \mathrm{~ms}$ during RVS, and $126 \pm 37 \mathrm{~ms}$ with BVS $(p<0.001)$. The left atrium diameter with RVS was $40 \pm 4 \mathrm{~mm}$, and $35 \pm 4 \mathrm{~mm}$ during BVS $(p<0.001)$. The end systolic and dyastolic LV diameters were respectivelly $49 \pm 13 \mathrm{~mm}$ and $59 \pm 11 \mathrm{~mm}$ during RVS, and $42 \pm 12 \mathrm{~mm}$ and $52 \pm 10 \mathrm{~mm}$ with BVS $(\mathrm{p}<0.001)$. The LV shortening fraction established by RVS was $18 \pm 7 \%$, and with BVS was $22 \pm 8 \%(\mathrm{p}=0.017)$. The LV ejection fraction during RVS was $33 \pm 14 \%$ and with BVS was $46 \pm 17 \%(p<0.001)$.

Conclusion: In the studied pattern, biventricular artificial stimulation determined a significant improvement of the hemodynamic performance in comparison to the right ventricular stimulation, and a QRS complex with duration close to the physiological.

DESCRIPTORS: Myocardial revascularization, postoperative period. Cardiac pacing, artificial. Pacemaker, artificial. 
Albuquerque L C, Sant'anna J R, Zago A J, Velho F J P, Petracco J B - Estudo comparativo das estimulações ventricular direita e biventricular no pós-operatório de revascularização miocárdica. Rev Bras Cir Cardiovasc 2002; 17(1): 61-72.

\section{REFERÊNCIAS BIBLIOGRÁFICAS}

1 Hochleitner $\mathrm{M}$, Hörtnagl $\mathrm{H}$, Ng C K, Hörtnagl $\mathrm{H}$, Gschnitzer F, Zechmann W - Usefulness of physiologic dual-chamber pacing in drug-resistant idiopathic dilated cardiomyopathy. Am J Cardiol 1990; 66:198-202.

2 Hochleitner M, Hörtnagl H, Hörtnagl H, Fridrich L, Gschnitzer F - Long-term efficacy of physiologic dualchamber pacing in the treatment of end-stage idiopathic dilated cardiomyopathy. Am J Cardiol 1992;70:1320-5.

3 Innes D, Leitch J W, Fletcher P J - VDD pacing at short atrioventricular intervals does not improve cardiac output in patients with dilated heart failure. Pacing Clin Electrophysiol 1994; 17: 959-65.

4 Linde C, Gadler F, Edner M, Nordlander R, Rosenqvist M, Rydén $\mathrm{L}$ - Results of atrioventricular synchronous pacing with optimized delay in patients with severe congestive heart failure. Am J Cardiol 1995; 75: 919-23.

5 Shinbane J S, Chu E, DeMarco T et al. - Evaluation of acute dual-chamber pacing with a range of atrioventricular delays on cardiac performance in refractory heart failure. J Am Coll Cardiol 1997; 30:1295-300.

6

Gold M R, Feliciano Z, Gottlieb S S, Fisher M L - Dualchamber pacing with a short atrioventricular delay in congestive heart failure: a randomized study. J Am Coll Cardiol 1995; 26: 967-73.

7 Bakker $\mathrm{P}$ A, Meijburg $\mathrm{H}$, de Jonge $\mathrm{N}$, Van Mechelen R, Wittkamp F, Mower M, et al. - Beneficial effects of biventricular pacing in congestive heart failure. Pacing Clin Electrophysiol 1994; 17: 820. [Abstract]

8 Cazeau S, Ritter P, Lazarus A et al. - Multisite pacing for end-stage heart failure: early experience. Pacing Clin Electrophysiol 1996; 19:1748-57.

9 Auricchio A, Ding J, Kramer A et al. - Comparison of left ventricular pacing sites for heart failure patients. Circulation 1998; 98: 302.

10 Leclercq C, Cazeau S, Le Breton H et al. - Acute hemodynamic effects of biventricular DDD pacing in patients with end-stage heart failure. J Am Coll Cardiol 1998; 32:1825-31.

11 Stellbrink C, Auricchio A, Diem B et al. - Potential benefit of biventricular pacing in patients with congestive heart failure and ventricular tachyarrhythmia. $A m \mathrm{~J}$ Cardiol 1999; 83(5B):143D-150D.

12 Alonso C, Leclercq C, Victor F et al. - Electrocardiographic predictive factors of long-term clinical improvement with multisite biventricular pacing in advanced heart failure. Am J Cardiol 1999; 84:1417-21.

13 Gras D, Mabo P, Tang T et al. - Multisite pacing as a supplemental treatment of congestive heart failure: preliminary results of the Medtronic Inc. InSync Study. Pacing Clin Electrophysiol 1998; 21: 2249-55.

14 Auricchio A, Klein H, Tockman B et al. - Transvenous biventricular pacing for heart failure: can the obstacles be overcome? Am J Cardiol 1999; 83(5B): 136D-142D.
15 Pachón Mateos J C, Albornoz R N, Pachón Mateos E I et al. - Estimulação ventricular direita bifocal no tratamento de miocardiopatia dilatada com insuficiência cardíaca. Arq Bras Cardiol 1999; 73: 485-98.

16 Saxon L A, Boehmer J P, Hummel J et al. - Biventricular pacing in patients with congestive heart failure: two prospective randomized trials. The VIGOR CHF and VENTAK CHF Investigators. Am J Cardiol 1999; 83(5B):120D-123D.

17 Auricchio A, Stellbrink C, Sack S et al. - The Pacing Therapies for Congestive Heart Failure (PATH-CHF) study: rationale, design, and endpoints of a prospective randomized multicenter study. $A m \mathrm{~J}$ Cardiol 1999; 83(5B):130D-135D.

18 Goldman L - Cost-effectiveness perspectives in coronary heart disease. Am Heart J 1990; 119(3Pt2): 733-40.

19 Del Rizzo D F, Fremes S E, Christakis G T, Sever J, Goldman B $S$ - The current status of myocardial revascularization: changing trends and risk factor analysis. J Card Surg 1996; 11:18-29.

20 Hartzler G O, Maloney J D, Curtis J J, Barnhorst D A - Hemodynamic benefits of atrioventricular sequential pacing after cardiac surgery. Am J Cardiol 1977; 40: 232-6.

21 Betocchi S, Piscione F, Villari B et al. - Effects of induced asynchrony on left ventricular diastolic function in patients with coronary artery disease. J Am Coll Cardiol 1993; 21: 1124-31.

22 Foster A H, Gold M R, McLaughlin J S - Acute hemodynamic effects of atrio-biventricular pacing in humans. Ann Thorac Surg 1995; 59: 294-300.

23 Waldo A L \& MacLean $\mathrm{W} \mathrm{A} \mathrm{H}$, eds. Diagnosis and treatment of cardiac arrhythmias following open heart surgery. New York: Futura Publishing; 1980.

24 Bernstein A D, Camm A J, Fletcher R D et al. - The NASPE/BPEG generic pacemaker code for antibradyarrhythmia and adaptive-rate pacing and antitachyarrhythmia devices. Pacing Clin Electrophysiol 1987; 10(4Pt1): 794-9.

25 Hatala R, Savard P, Tremblay G et al. - Three distinct patterns of ventricular activation in infarcted human hearts. An intraoperative cardiac mapping study during sinus rhythm. Circulation 1995; 91: 1480-94.

26 Xiao H B, Brecker S J, Gibson D G - Effects of abnormal activation on the time course of the left ventricular pressure pulse in dilated cardiomyopathy. Br Heart $J$ 1992; 68: 403-7.

27 Grines C L, Bashore T M, Boudoulas H, Olson S, Shafer $\mathrm{P}$, Wooley C F - Functional abnormalities in isolated left bundle branch block. The effect of interventricular asynchrony. Circulation 1989; 79: 845-53.

28 Forfang K, Otterstad J E, Ihlen H - Optimal atrioventricular delay in physiological pacing determined by Doppler echocardiography. Pacing Clin Electrophysiol 1986; 9(1Pt1): 17-20.

29 Vanoverschelde J L, Wijns W, Michel X, Cosyns J, Detry $J \mathrm{M}$ - Asynchronous (segmental early) relaxation impairs left ventricular filling in patients with coronary artery disease and normal systolic function. J Am Coll Cardiol 1991; 18: 1251-8. 